\title{
Rolipram plays an anti-fibrotic effect in ligamentum flavum fibroblasts by inhibiting the activation of ERK $1 / 2$
}

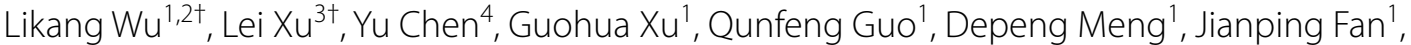 \\ Guoqiang Song ${ }^{2^{*}}$ and Peng $\mathrm{Xu}^{1,5^{*}}$
}

\begin{abstract}
Background: Fibrosis is an important factor and process of ligamentum flavum hypertrophy. The expression of phosphodiesterase family (PDE) is related to inflammation and fibrosis. This article studied the expression of PDE in hypertrophic ligamentum flavum fibroblasts and investigated whether inhibition of PDE4 activity can play an antifibrotic effect.
\end{abstract}

Methods: Samples of clinical hypertrophic ligamentum flavum were collected and patients with lumbar disc herniations as a control group. The collagenase digestion method is used to separate fibroblasts. qPCR is used to detect the expression of PDE subtypes, type I collagen (Col I), type III collagen (Col III), fibronectin (FN1) and transforming growth factor $\beta 1$ (TGF- $\beta 1$ ). Recombinant TGF- $\beta 1$ was used to stimulate fibroblasts to make a fibrotic cell model and treated with Rolipram. The morphology of the cells treated with drugs was observed by Sirius Red staining. Scratch the cells to observe their migration and proliferation. WB detects the expression of the above-mentioned multiple fibrotic proteins after drug treatment. Finally, combined with a variety of signaling pathway drugs, the signaling mechanism was studied.

Results: Multiple PDE subtypes were expressed in ligamentum flavum fibroblasts. The expression of PDE4A and 4B was significantly up-regulated in the hypertrophic group. Using Rolipram to inhibit PDE4 activity, the expression of Col I and TGF- $\beta 1$ in the hypertrophic group was inhibited. Col I recovered to the level of the control group. TGF- $\beta 1$ was significantly inhibited, which was lower than the control group. Recombinant TGF- $\beta 1$ stimulated fibroblasts to increase the expression of Col I/III, FN1 and TGF- $\beta 1$, which was blocked by Rolipram. Rolipram restored the increased expression of $\mathrm{p}$-ERK1/2 stimulated by TGF- $\beta 1$.

Conclusion: The expressions of PDE $4 \mathrm{~A}$ and $4 \mathrm{~B}$ in the hypertrophic ligamentum flavum are increased, suggesting that it is related to the hypertrophy of the ligamentum flavum. Rolipram has a good anti-fibrosis effect after inhibiting the activity of PDE4. This is related to blocking the function of TGF- $\beta 1$, specifically by restoring normal ERK $1 / 2$ signal.

Keywords: Rolipram, Phosphodiesterase, Ligamentum Flavum hypertrophy, ERK1/2, Fibrosis, TGF- $\beta 1$

\footnotetext{
*Correspondence: sgq@cczu.edu.cn; xupengspine@163.com

${ }^{\dagger}$ Likang Wu and Lei Xu contributed equally to this work.

2 School of Pharmacy \& School of Medicine, ChangZhou University,

Changzhou 213164, People's Republic of China

${ }^{5}$ Institute of Bioinformatics and Medical Engineering, School of Electrical

and Information Engineering, Jiangsu University of Technology,

Changzhou 213001, People's Republic of China

Full list of author information is available at the end of the article
}

(c) The Author(s) 2021. Open Access This article is licensed under a Creative Commons Attribution 4.0 International License, which permits use, sharing, adaptation, distribution and reproduction in any medium or format, as long as you give appropriate credit to the original author(s) and the source, provide a link to the Creative Commons licence, and indicate if changes were made. The images or other third party material in this article are included in the article's Creative Commons licence, unless indicated otherwise in a credit line to the material. If material is not included in the article's Creative Commons licence and your intended use is not permitted by statutory regulation or exceeds the permitted use, you will need to obtain permission directly from the copyright holder. To view a copy of this licence, visit http://creativecommons.org/licenses/by/4.0/. The Creative Commons Public Domain Dedication waiver (http://creativeco mmons.org/publicdomain/zero/1.0/) applies to the data made available in this article, unless otherwise stated in a credit line to the data. 


\section{Introduction}

Hypertrophy of the ligamentum flavum (LF) is an important factor in lumbar spinal stenosis, causing pain and difficulty walking [1]. Surgery is currently the main treatment method, but it is traumatic and difficult [2]. People hope to explore the causes of LF hypertrophy and find safe and efficient treatment strategies. In the process of LF hypertrophy, abnormal mechanical stress stimulates chronic inflammation and changes in the tissue microenvironment [3]. Infiltrating macrophages secrete a large number of inflammatory factors and activate fibroblasts [4]. Stimulates the secretion and deposition of collagen fibers, which eventually causes the LF hypertrophy [5]. Therefore, inflammation and fibrosis are two important factors for LF hypertrophy [6]. Substances that exert anti-inflammatory and antifibrotic effects may be beneficial to the treatment of LF hypertrophy.

Phosphodiesterase (PDE) is widely expressed in organisms. Changes in its expression and activity are related to inflammation and fibrosis [7]. In multiple reports, inhibiting PDE activity has played an antifibrotic effect. For example, PDE4 inhibitors can antagonize fibroblast activation, thereby improving renal fibrosis [8] and inhibit fibroblast-mediated contraction of three-dimensional collagen gels in lung fibrosis [9]. In this study, a number of clinical samples of the LF were collected to detect differences in the expression of PDE subtypes in the two fibroblasts. Explore the relationship between the expression of PDE subtypes and LF hypertrophy. Then, we conducted further research on the increased expression of PDE4A and 4B. The specific inhibitor rolipram was used to inhibit the activity of PDE4A and 4B to investigate the anti-fibrosis effect.

\section{Materials and methods}

\section{Specimens}

This study was approved by Committee on Ethics of Biomedicine, Second Military Medical University (Shanghai, China). Lumbar spine 4/5 segment LF samples were collected from 25 patients who underwent lumbar surgery at Shanghai Changzheng Hospital from June 2019 to December 2019. A total of 15 patients with definite LF hypertrophy constituted the hypertrophy group (LFH) and 10 patients with lumbar disc herniation or trauma constituted the control group (NLFH). LF thickness was measured at the facet joint level by T1-weighted magnetic resonance (MR) imaging.

\section{Compounds and drugs}

Rolipram (CAS: 61413-54-5, CSNpharm, Shanghai, China), WAY-262611 (CAS: 1123231-07-1, CSNpharm),
LY294002 (CAS: 154447-36-6, CSNpharm), SCH772984 (CAS: 942183-80-4, CSNpharm) were dissolved in DMSO. It was diluted 1000-10,000 times with complete medium before use and the final concentration in media was $0.01-1 \mu \mathrm{M}$. Rh-TGF- $\beta 1$ (CST, \#8915, USA) was dissolved in $20 \mathrm{mM}$ citrate with a $\mathrm{pH}=3.0$ according instructions provided by the manufacturer. The final concentration was $5 \mathrm{ng} / \mathrm{mL}$.

\section{LF fibroblast isolation and culture}

LF tissue samples harvested from lumbar spinal stenosis patients were washed in physiological saline, minced and incubated for $1 \mathrm{~h}$ at $37^{\circ} \mathrm{C}$ in Dulbecco's Modified Eagle Medium (DMEM; HyClone, USA) containing 0.2\% type I collagenase (Sigma, USA). The suspension was filtered using a $70 \mathrm{~mm}$-mesh cell strainer (Falcon, BD, USA) and cells were seeded into the wells of a $60 \mathrm{~mm}$ culture dish (NEST, Wuxi, China) containing DMEM supplemented with $10 \%$ fetal bovine serum (FBS; Transgen, Beijing, China), $100 \mathrm{U} / \mathrm{mL}$ penicillin and $100 \mathrm{pg} / \mathrm{mL}$ streptomycin (HyClone). Subsequent experiments were performed using cells that were passaged between 2 and 5 times.

\section{RNA isolation and quantitative RT-PCR (qRT-PCR)}

Total RNA was isolated from samples or cells using the Total RNA Isolation Kit (Vazyme, Nanjing, China). Next, cDNA was reverse transcribed from isolated RNA by incubating $1 \mu \mathrm{g}$ of DNase treated RNA with components of a first-strand synthesis kit (Promega, USA). Real-time PCR was performed using TB Green ${ }^{\circledR}$ Premix Ex Taq ${ }^{\mathrm{TM}}$ (TAKARA, Japan) in a thermal cycler with the following parameters: 40 cycles, $95^{\circ} \mathrm{C}$ for $10 \mathrm{~s}, 60^{\circ} \mathrm{C}$ for $30 \mathrm{~s}$. All primers (Table 1) were synthesized by Qingke Inc. (Shanghai, China). Data were analyzed using the $2^{\wedge-\Delta \Delta C T}$ method to compare gene expression levels.

\section{Western blot analysis}

Tissue specimens and cells were lysed using RIPA buffer containing a protease inhibitors cocktail (Epizyme, Shanghai, China). Lysates were heated in $95^{\circ} \mathrm{C}$ for $10 \mathrm{~min}$ in protein sample loading buffer (Epizyme). Total cell lysates were separated using 10\% SDS-PAGE (Epizyme) and transferred to an Immobilon-P Transfer Membrane (Millipore, USA). Membranes were blocked using 5\% nonfat milk dissolved in Tris-buffered saline and then incubated with a FN1 (CST, \#26836, USA), ERK1/2 (CST, \#4695, USA), p-ERK1/2 (CST, \#4370, USA), TGF- $\beta$ (CST, \#3711, USA), COL3A1 (CST, \#30565, USA), collagen I (Abcam, ab34170, USA) and $\alpha$-SMA (Servicebio, GB11044, China) antibodies. The following day, membranes were incubated with a horseradish peroxidaseconjugated secondary antibody (Transgen). GAPDH 
Table 1 Forward and reverse primer sequences for LF hypertrophy markers and PDE isoforms used in qRT-PCR

\begin{tabular}{|c|c|c|}
\hline Gene & Forward primer & Reverse prime \\
\hline PDE1A & TGAAGGGATTGACAGAGC & ATGGTCCACCGATAATGC \\
\hline PDE1B & CGCCGAGCAGAGGAGAAG & AGAACCGCAGTAGAGTAAGTGG \\
\hline PDE1C & $\begin{array}{l}\text { TGGAAGTGGGATACAGCA } \\
\text { AGC }\end{array}$ & CTCCGTCAGCCAGTTCGC \\
\hline PDE2A & $\begin{array}{l}\text { ATCTTTGCCTTGTTTATT } \\
\text { TCCTG }\end{array}$ & CAGCCAGCACAGATTTCG \\
\hline PDE3A & $\begin{array}{l}\text { GATGATAAATACGGATGT } \\
\text { CTGTC }\end{array}$ & ACCGCCTGAGGAGCACTAG \\
\hline PDE3B & TGCCTTCTTCTTCCTCACCTG & GACCACCACTGCCACACC \\
\hline PDE4A & TTCACGGACCTGGAGATTC & TGAGGAACTGGTTGGAGAC \\
\hline PDE4B & $\begin{array}{l}\text { CAAGCCTAAACAATACAA } \\
\text { GCATC }\end{array}$ & $\begin{array}{l}\text { TGAGAATATCCAGCCACATTA } \\
\text { AAG }\end{array}$ \\
\hline PDE4C & CACCTGGCTGTGGGCTTC & ACTCAGTCGCTGCTTGGC \\
\hline PDE4D & $\begin{array}{l}\text { CTACTGGCTGATTTGAAG } \\
\text { ACTATG }\end{array}$ & GCTGGAGAGGCTTTGTTGG \\
\hline PDE5A & $\begin{array}{l}\text { ATCAGGAAACGGTGGGAC } \\
\text { ATTTAC }\end{array}$ & $\begin{array}{l}\text { CTTGTTCTCCAGCAGTGAAGT } \\
\text { CTC }\end{array}$ \\
\hline PDE7A & AGATAGGTGCTCTGATACTAG & ATGTCTGTGTCTGGTGTC \\
\hline PDE7B & GGCTTCTTGCTCATTTGC & CCTGTTGATGTCTGTTGC \\
\hline PDE8A & ATGTTTGCTCGCTTTGGAATC & $\begin{array}{l}\text { CAGAATGTGTAGAATTGTGGT } \\
\text { AGG }\end{array}$ \\
\hline PDE8B & CAAATCCCTCCGAGCACAC & CTCCATAAATCTCCTGTTGAAGC \\
\hline PDE9A & $\begin{array}{l}\text { CGTGGAATTGGAAGGACT } \\
\text { AAAAG }\end{array}$ & GAGTCAACTTCTTGTGGTTATCC \\
\hline PDE10A & AACTATCGGCGGGTTCCTTATC & $\begin{array}{l}\text { GCGTGTGATTGTTCTGAAGTA } \\
\text { TGG }\end{array}$ \\
\hline PDE11A & CTGCTGGGTTTCAAGACATTC & GCTTGGAAGGCATTGTTGG \\
\hline TGF- $\beta 1$ & CTGTACATTGACTTCCGCAAG & TGTCCAGGCTCCAAATGTAG \\
\hline Coll & AAAGATGGACTCAACGGTCTC & CATCGTGAGCCTTCTCTTGAG \\
\hline Col III & CGCCCTCCTAATGGTCAAGG & TTCTGAGGACCAGTAGGGCA \\
\hline$\beta$-actin & GCGGGAAATCGTGCGTGACA & $\begin{array}{l}\text { GGAAGGAAGGCTGGAAGA } \\
\text { GTGC }\end{array}$ \\
\hline
\end{tabular}

(Transgen) and $\beta$-tubulin (Transgen) were used as loading controls. Finally, membranes were visualized using an ECL western blotting detection reagent (Epizyme).

\section{Statistics and heat map drawing}

Statistical analyses and graphs were performed and generated using GraphPad Prism 8 Software. Differences in the fold change between patients and controls were assessed using an independent-samples t test. A $P$ value less than 0.05 was considered as statistically significant. Use GraphPad software to draw the heat map. The shade of the color in the figure represents the $\triangle \triangle \mathrm{Ct}$ value of the qPCR result.

\section{Results}

\section{The expression of PDE subtypes in the LF fibroblast}

After extracting total RNA from 25 samples, a qPCR reaction was performed and the results were statistically summarized. Hypertrophic group and control constituted the expression of PDE family in fibroblasts (Fig. 1A). Among the investigated PDE subtypes, PDE1B, $2 \mathrm{~A}, 3 \mathrm{~B}, 4 \mathrm{C}, 7 \mathrm{~B}, 8 \mathrm{~B}, 9 \mathrm{~A}$ and $11 \mathrm{~A}$ are expressed in very little or almost no expression in LF fibroblasts. The expression levels of PDE4A and PDE4B in the hypertrophic group (LFH) were significantly higher than those in the control group (NLFH) $(P<0.01$, Fig. 1B, D). The expression levels of other PDE subtypes also vary among individuals, but there is no statistical difference (Table 2). In addition, the expression of PDE4A and PDE4B increased as the thickness of LF increased (Fig. 1C, E).

\section{The relationship between the up-regulation of PDE4A and $B$ and fibrosis markers}

We also tested the expression of markers related to LF hypertrophy. The expressions of Col I, Col III, FN1 and TGF- $\beta 1$ were up-regulated in the hypertrophy group (Fig. 2A-D). The mRNA expression of Col I, FN1 has a linear correlation with the thickness of the LF (Fig. 2E, F). Comparing the relationship between the expression of PDE4A and 4B and the expression of Col I, the expression of PDE4B and Col I has a linear correlation $(\mathrm{R}=0.5947$, $P<0.01$, Fig. 2G). The linear correlation between the expression of PDE4A and Col1 is shown in the Fig. $2 \mathrm{H}$.

\section{Rolipram inhibits the expression of col I and TGF- $\beta 1$ in hypertrophic fibroblasts}

Rolipram can inhibit the activity of all PDE4 subtypes. Compared with normal LF fibroblasts, the expression of Col I and TGF- $\beta 1$ increased in the hypertrophic group (Fig. 3A, B). After Rolipram inhibited the activity of PDE4 in hypertrophic LF fibroblasts, the expression levels of Col I and TGF- $\beta 1$ decreased. The expression of TGF- $\beta 1$ dropped to a very low level after Rolipram treatment, which was lower than that of the control group. The expression of Col I in the hypertrophy group was downregulated after Rolipram treatment, which was tended to the expression level of Col I in the control group.

\section{Rolipram blocks fibroblast fibrosis stimulated by TGF- $\beta 1$}

Fibroblasts have enhanced proliferation and migration ability after stimulation by recombinant human TGF$\beta 1$. The morphology of the cell changes, from a spindle shape to a polygonal shape. The edges of the cells are blurred (Fig. 4A, B). The expression of fibrosis markers such as Col I, Col III, FN1 and $\alpha$-SMA was up-regulated (Fig. 4C-F). Under the stimulation of exogenous TGF$\beta 1$, the expression of endogenous TGF- $\beta 1$ in fibroblasts increases (Fig. 4G). After administration of Rolipram, the above-mentioned fibrotic protein expression was restored. The aggregation of fibroblasts was suppressed, but polygonal fibroblasts were still visible (Fig. 4A.d). 


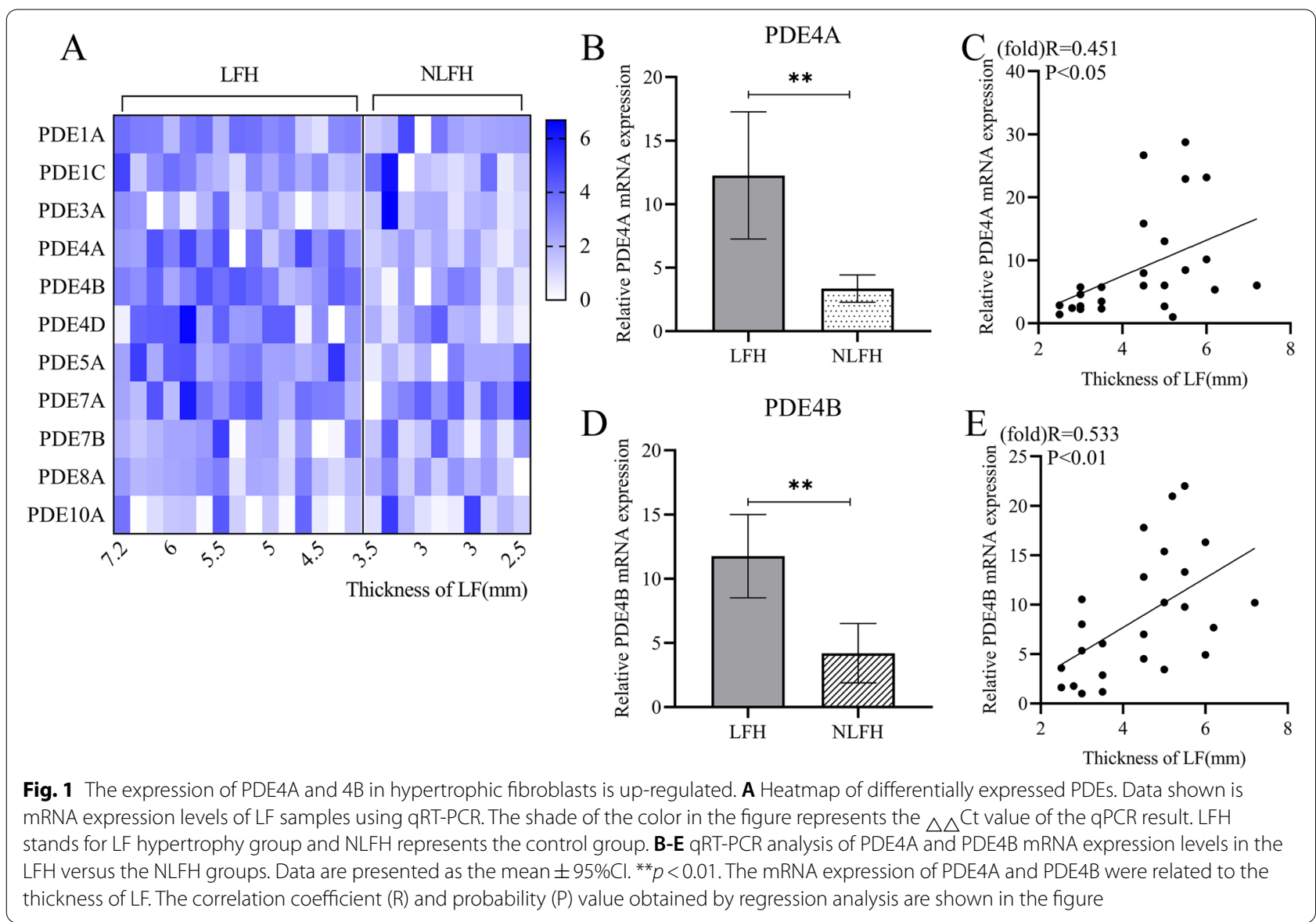

Table 2 Correlation between gene in Fig. 1A mRNA expression and LF thickness

\begin{tabular}{lll}
\hline Gene name & R value & P value \\
\hline PDE1A & 0.2696 & 0.1924 \\
PDE1C & 0.05952 & 0.7775 \\
PDE3A & -0.1015 & 0.6292 \\
PDE4A & 0.4510 & $0.0237^{*}$ \\
PDE4B & 0.5335 & $0.006^{* *}$ \\
PDE4D & 0.3137 & 0.1268 \\
PDE5A & 0.3074 & 0.1349 \\
PDE7A & -0.1082 & 0.6066 \\
PDE7B & 0.03061 & 0.8845 \\
PDE8A & 0.1679 & 0.4225 \\
PDE10A & -0.08561 & 0.6841 \\
TGF- $\beta 1$ & 0.4484 & $0.0246^{*}$ \\
ColI & 0.6745 & $0.0002^{* * *}$ \\
Col III & 0.5051 & $0.01^{*}$ \\
\hline
\end{tabular}

\section{The signaling pathway is involved in Rolipram's} anti-fibrosis effect

The fibroblasts stimulated by TGF- $\beta 1$ were treated with four drugs and the expression of TGF- $\beta 1$ in the fibroblasts was detected. The results showed that Rolipram reduced the expression of TGF- $\beta 1$ (Fig. 5A. a). Activation of the $\beta$-catenin pathway increases the expression of TGF- $\beta 1$ (Fig. 5A. b). Inhibiting the signal transduction of ERK1/2 and PI3K showed the effect of inhibiting the expression of TGF- $\beta 1$ (Fig. $5 \mathrm{~A}$. c, d). We conduct in-depth research on the ERK1/2 pathway. The use of Rolipram and ERK1/2 inhibitor SCH772984 both reduced the expression of TGF- $\beta 1$ and FN1 (Fig. 5B). After fibroblasts stimulated by TGF- $\beta 1$, the expression of p-ERK1/2 increased, which was inhibited by Rolipram (Fig. 5C).

\section{Discussion}

The dysregulation of PDE expression is related to many diseases [10]. We found that the mRNA expression of PDE4A and $4 \mathrm{~B}$ was up-regulated in the hypertrophic group (LFH). The up-regulated PDE4A and 4B inactivate intracellular cAMP and cause a variety of signal pathway 

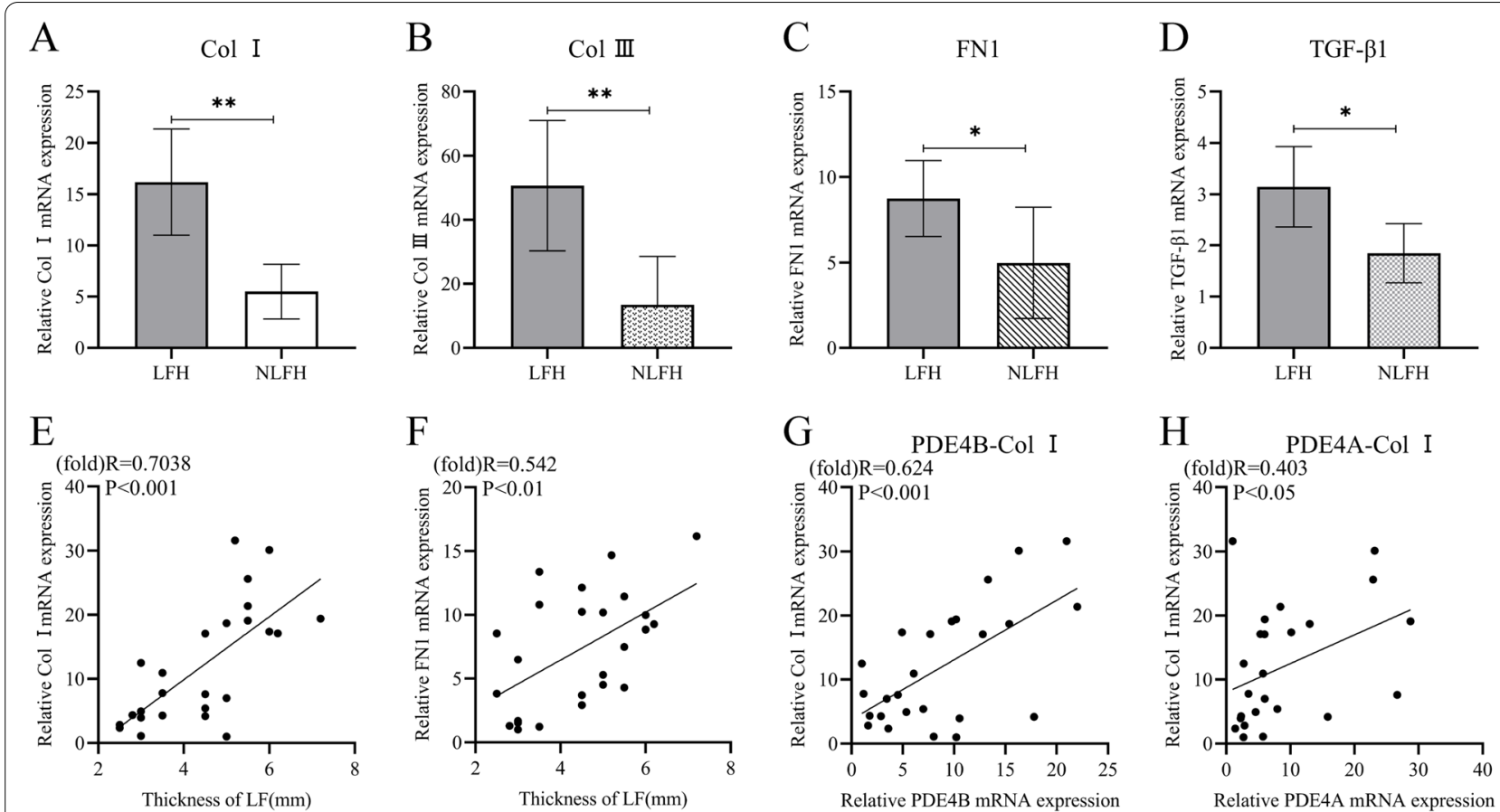

Fig. 2 The expression of PDE $4 A$ and $4 B$ is related to fibrosis. The histogram results in $\mathbf{A}, \mathbf{B}, \mathbf{C}$ and $\mathbf{D}$ were presented as the mean $\pm 95 \% \mathrm{Cl}$. ${ }^{*} p<0.05$, ${ }^{* *} p<0.01$. E The Col I mRNA expression was related to the thickness of LF. F There is a linear correlation between FN1 mRNA expression and LF thickness. G-H The mRNA expression of PDE4A and PDE4B were related to the Col I

changes. In diethylnitrosamine-induced liver fibrosis, the overexpression of PDE4 is an important pathogenic factor. Enhancing CAMP and CREB levels by inhibiting PDE4 activity can regulate inflammation and fibrosis, thereby exerting a therapeutic effect [11]. In our results, the expression of PDE4B increased linearly with the expression of Col I. Col I is an important marker of fibrosis and LF hypertrophy [12]. This means that increased expression of PDE4B is related to fibrosis. Not only that, the expression of PDE4A and PDE4B increased linearly with the increase of the thickness of the LF. This implies that the increased expression of PDE4A and $4 \mathrm{~B}$ is related to LF hypertrophy.

We used Rolipram, an inhibitor of PDE4A and 4B, to treat LF fibroblasts to investigate the effect of inhibiting PDE4 activity. In the hypertrophic LF, the expression of a variety of cytokines increases. Including TNF- $\alpha$, IL- $1 \beta$ and IL- 6 related to inflammation, TGF- $\beta 1$, CTGF, Col I and Col III related to fibrosis [13]. After treating hypertrophic fibroblasts with Rolipram, the expression of Col I and endogenous TGF- $\beta 1$ was down-regulated. And the expression of TGF- $\beta 1$ was lower than that of the control group (Fig. 3). This indicates that Rolipram can significantly inhibit the expression of TGF- $\beta 1$ in fibroblasts. Rolipram increases the content of cAMP in cells and stimulates the activation of downstream PKA and CREB, thereby inhibiting the function of TGF- $\beta 1$ [14].

TGF- $\beta 1$ is up-regulated in hypertrophic LF tissue and is one of the important factors leading to LF hypertrophy [15]. TGF- $\beta 1$ antibodies and their inhibitors have been proven to be effective in treating certain fibrotic diseases by targeting the TGF- $\beta 1$ signal transduction pathway [16]. For example, CCN5 protein inhibits the differentiation of fibroblasts induced by TGF- $\beta 1$ into myofibroblasts, reducing fibrosis and LF hypertrophy [17]. Down-regulation of cytokine receptor-like factor 1 (CRLF1) reduces the fibrosis caused by inflammatory cytokines and mechanical stress by blocking the TGF- $\beta 1$ pathway. In mouse models, knocking out CRLF1 can prevent the formation of LF hypertrophy [18]. In our experiment, Rolipram's anti-fibrosis effect may be related to the blocking of TGF- $\beta 1$ signal transduction, and we have conducted in-depth research on this.

There is a large gap between samples of primary fibroblasts. The expression of fibrotic proteins such as TGF- $\beta 1$ and Col I is generally increased in the hypertrophic group, but there is a large gap in a specific single sample. TGF- $\beta 1$ can promote the synthesis of extracellular matrix (ECM) protein and stimulate the proliferation and migration of fibroblasts [19]. Using 

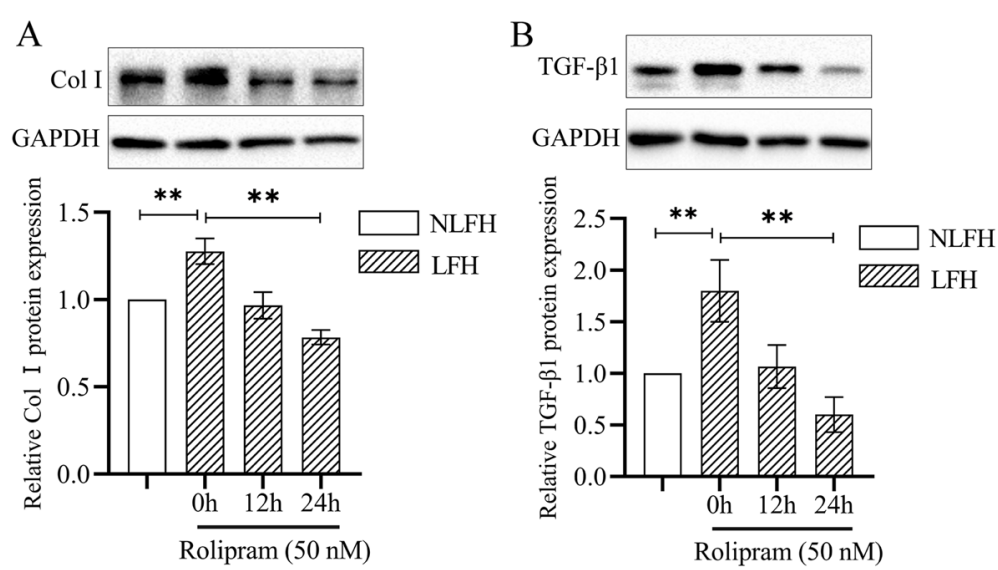

Fig. 3 Inhibiting PDE4 with Rolipram attenuates Col I and TGF- $\beta 1$ expression levels in LF hypertrophy fibroblasts. Primary LF fibroblasts were treated with rolipram $(50 \mathrm{nM})$ at different times. Western blot analysis shows the expression for Col I and TGF- $\beta 1$ in LF fibroblasts treated with Rolipram. GAPDH served as an endogenous control. ${ }^{* *} P<0.01$

recombinant TGF- $\beta 1$ to stimulate fibroblasts to form a cell model of fibrosis, the expression of fibrotic protein is relatively stable, which is suitable for subsequent mechanism research. After stimulation by recombinant TGF- $\beta 1$, the expression of a variety of fibrotic proteins increased, including Col I, Col III, FN1 and endogenous TGF- $\beta 1$ (Fig. 4). FN1 plays an important role in the process of fibrosis and can affect the function of TGF- $\beta 1$. In tissues, isolated TGF- $\beta 1$ and ECM inhibit the activation of TGF- $\beta 1$, thereby limiting its ability to stimulate cell surface receptors [20]. FN1 can also be used as a bridge to transport TGF- $\beta 1$ and promote its function [21]. In addition, FN1 has increased expression in the hypertrophic LF. This means that the expression of FN1 is related to LF hypertrophy. After TGF- $\beta 1$ stimulation, fibroblasts changed their morphology, increased their proliferation and migration capabilities (Fig. 4). There are similar results in the Hur's report [22]. As an important marker of LF hypertrophy, TGF- $\beta 1$ promotes fibrosis and causes tissue hypertrophy of the LF. The expression of these up-regulated fibrotic proteins was down-regulated after Rolipram treatment (Fig. 4). Rolipram exerts a certain anti-fibrosis effect.

The TGF- $\beta$ family plays a key role in tissue fibrosis and mediates fibrosis through Smad-dependent or non-Smad pathways [23]. A variety of signaling pathways are related to TGF- $\beta 1$ to stimulate fibrosis, including $\beta$-catenin, PI3K and ERK1/2 [24]. When drugs were used to interfere with the three signal transductions, the function of TGF$\beta 1$ changed to varying degrees (Fig. 5). We conducted a detailed study on ERK1/2 signal transduction. When TGF- $\beta 1$ stimulates fibroblast activation, ERK1/2 is activated and the expression of p-ERK1/2 increases (Fig. 5). The activated ERK1/2 may affect the activity of PDE4 [10]. The up-regulated PDE4A and 4B deactivate cAMP hydrolysis and promote the process of fibrosis. The use of ERK1/2 pathway inhibitors or Rolipram can block the fibrosis stimulated by TGF- $\beta 1$. Rolipram exerts an antifibrotic effect by restoring normal p-ERK $1 / 2$ expression.

In conclusion, we found that the expression of PDE4A and 4B mRNA in the hypertrophic LF is upregulated, which is importantly related to the hypertrophy of the LF. Inhibiting the activity of PDE4A and 4B by Rolipram can inhibit a variety of fibrosis-related proteins and block the effect of TGF- $\beta 1$ by restoring the expression of $\mathrm{p}$-ERK $1 / 2$. This effect may be effective in treating LF hypertrophy.

\section{Abbreviations}

LF: Ligamentum flavum; PDE: Phosphodiesterase; Col I: Collagen I; Col III: Collagen III;TGF- $\beta 1$ : Transforming growth factor $\beta 1$; ERK1/2: Extracellular regulated protein kinases 1/2; FN1: Fibronectin; CAMP: Cyclic adenosine monophosphate; ECM: Extracellular matrix; CREB: CAMP-response element binding protein; CRLF1: Cytokine receptor-like factor 1.

(See figure on next page.)

Fig. 4 Rolipram blocks TGF- $\beta 1$-induced LF fibroblast fibrosis. (A) a and b, Picture of fibroblasts (40x). c and d, staining with Sirius Red makes the fibroblasts easy to observe (100x). (B) Cell scratch test in LF fibroblasts (100x). (C-G) After fibroblasts were stimulated by TGF- $\beta 1$ and treated with Rolipram, the expression of Col I, Col3A1, FN1, a-SMA and TGF- 31 . GAPDH or $\beta$-tubulin served as an endogenous loading control. ${ }^{*} p<0.05$, ${ }^{* *} p<0.01,{ }^{* * *} p<0.001$ 


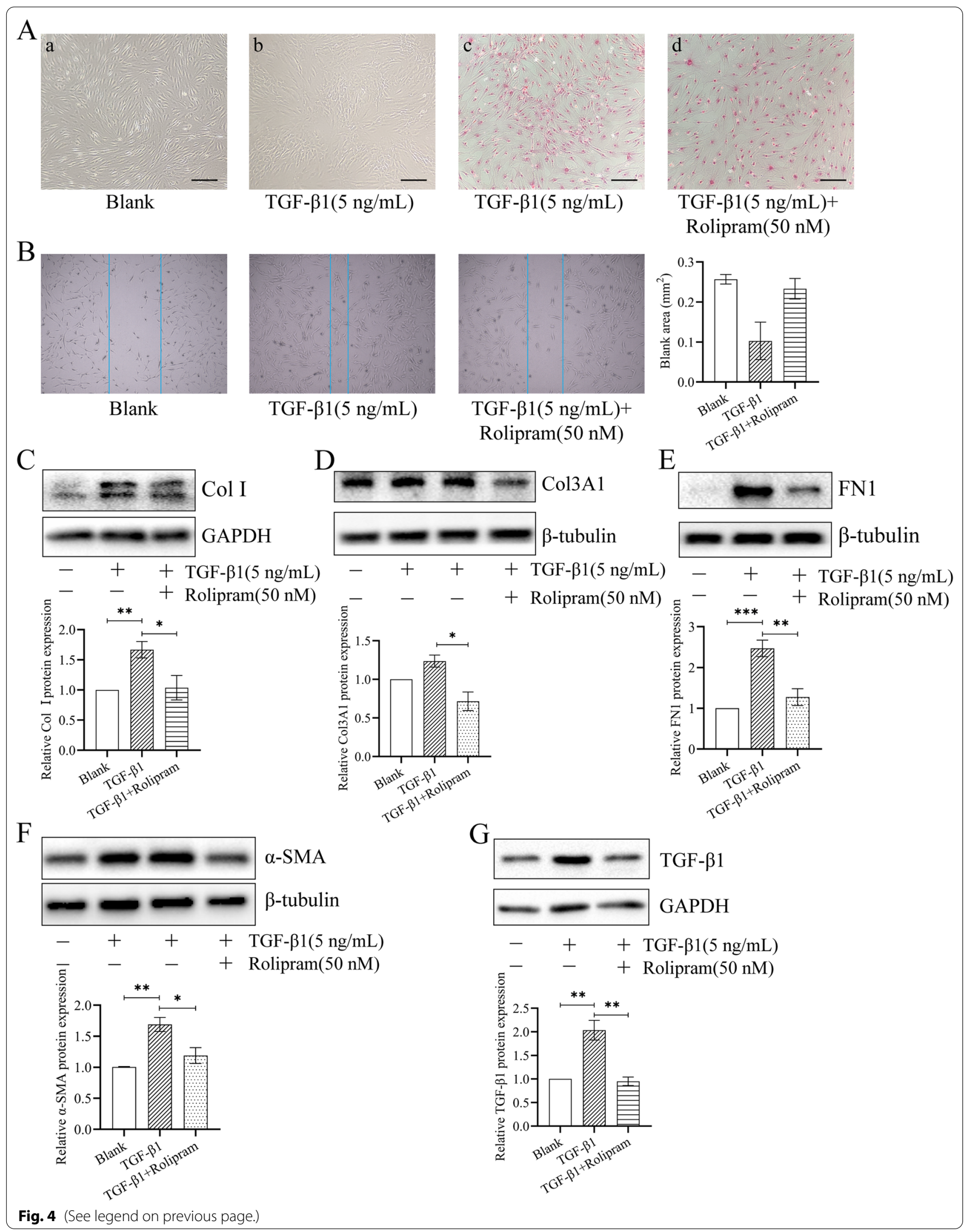



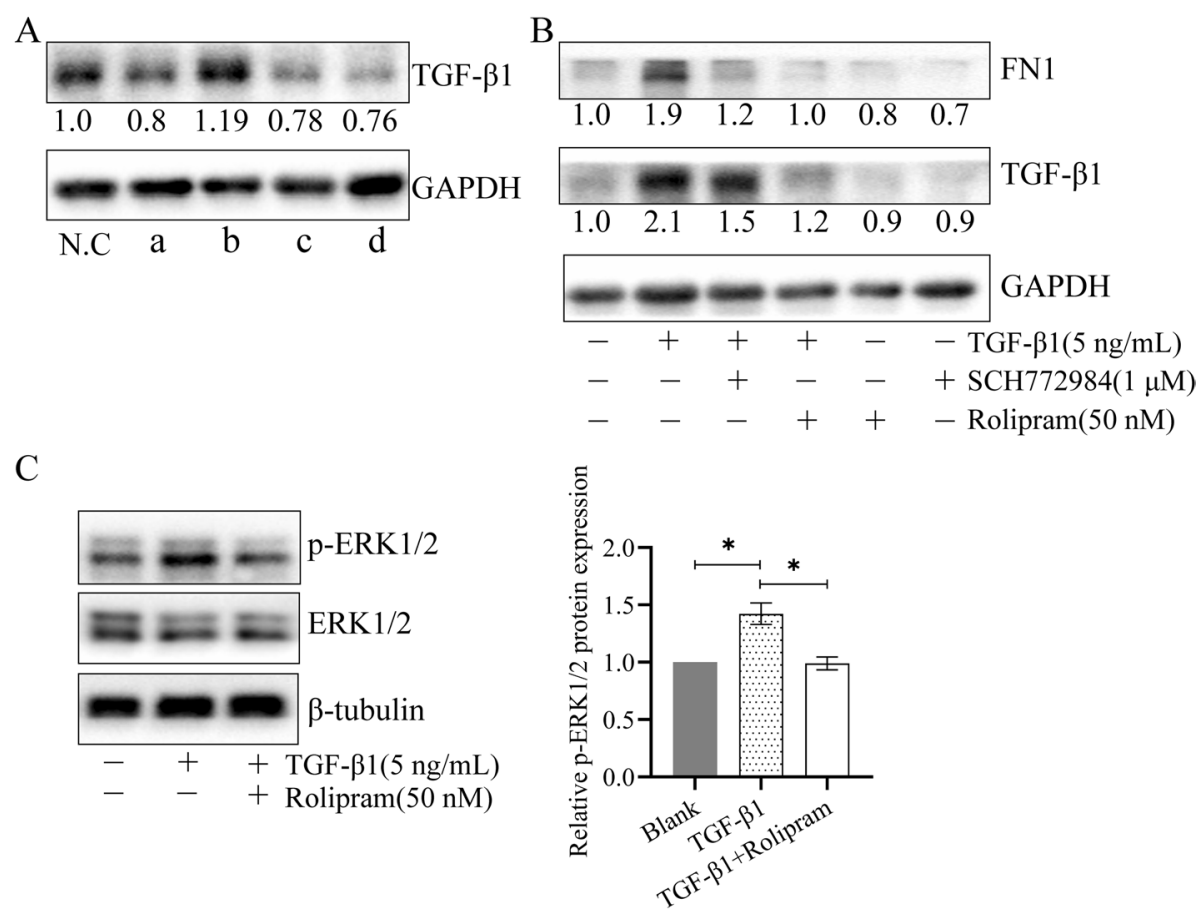

Fig. 5 Rolipram restores ERK1/2 signaling to block TGF- $\beta 1$ stimulated LF fibroblast fibrosis. (A) Different drugs were used to treat fibroblasts stimulated by TGF- $\beta 1$. WB detects the expression of TGF- $\beta 1$. a, Rolipram (50 nM) is a PDE4 inhibitor.; b, WAY-262611 (1 $\mu$ M) is the Wnt/ $\beta$-catenin

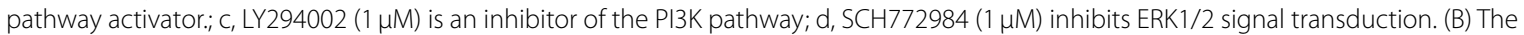
expression of FN1 and TGF- $\beta 1$ was up-regulated after exogenous TGF- $\beta 1$ stimulation. Using Rolipram or SCH772984 blocked this change. (C) Western blot analysis reveals the expression levels of p-ERK1/2 and ERK1/2 in LF fibroblasts treated with TGF- $\beta 1$ ( $5 \mathrm{ng} / \mathrm{mL}$ ) and Rolipram (50 nM, 24h). $\beta$-tubulin served as an endogenous loading control. ${ }^{*} p<0.05$

\section{Supplementary Information}

The online version contains supplementary material available at https://doi. org/10.1186/s12891-021-04712-9.

\section{Additional file 1.}

\section{Acknowledgements}

Not applicable.

\section{Authors' contributions}

XP and SG conceived of the design of the study. CY, XG and GQ participated in Clinical specimen and data collection. MD and FJ performed the statistical analysis. WL participated in cell experiment. WL and XL were a major contributor in writing the manuscript. All authors read and approved the final manuscript.

\section{Funding}

This work was supported by the National Natural Science Foundation of China (Nos. 81702184), Shanghai Municipal Commission of Health and Family Planning (Nos. 201740174) and Natural Science Foundation of Jiangsu Province (Nos. BE2019650).

\section{Availability of data and materials}

The datasets used and/or analyzed during the current study are available from the corresponding author upon reasonable request.

\section{Declarations}

\section{Ethics approval and consent to participate}

This study was approved by Committee on Ethics of Biomedicine, Second Military Medical University (Shanghai, China) and all methods were performed in accordance with the Ethics Committee guidelines. All patients gave written informed consent before participation in this study.

\section{Consent for publication}

Not applicable.

\section{Competing interests}

The authors declare that they have no competing interests.

\section{Author details}

'Department of Orthopedics, Changzheng Hospital, Naval Medical University, Shanghai 200003, People's Republic of China. ${ }^{2}$ School of Pharmacy \& School of Medicine, ChangZhou University, Changzhou 213164, People's Republic of China. Institute of Bioinformatics and Medical Engineering, School of Electrical and Information Engineering, Jiangsu University of Technology, Changzhou 213001, People's Republic of China. ${ }^{4}$ Department of Orthopedics, First Clinical Medical College, Shandong University of Traditional Chinese Medicine, Jinan 250355, People's Republic of China. ${ }^{5}$ Institute of Bioinformatics and Medical Engineering, School of Electrical and Information Engineering, Jiangsu University of Technology, Changzhou 213001, People's Republic of China.

Received: 31 October 2020 Accepted: 8 September 2021 Published online: 23 September 2021 


\section{References}

1. Salimi H, Suzuki A, Habibi H, et al. Biglycan expression and its function in human ligamentum flavum. Sci Rep. 2021;1(11):4867.

2. Zhu X, Qiu Z, Liu Z, et al. CT-guided percutaneous lumbar Ligamentum Flavum release by needle knife for treatment of lumbar spinal stenosis: a case report and literature review. J Pain Res. 2020;13:2073-81.

3. Sidon E, Shemesh S, Mor-Yossef Moldovan L, et al. Molecular profile of ultrastructure changes of the ligamentum flavum related to lumbar spinal canal stenosis. J Cell Biochem. 2019. https://doi.org/10.1002/jcb. 28451.

4. Saito $T$, Hara M, Kumamaru $H$, et al. Macrophage infiltration is a causative factor for Ligamentum Flavum hypertrophy through the activation of collagen production in fibroblasts. Am J Pathol. 2017;12(187):2831-40.

5. Chen J, Liu Z, Zhong G, et al. Hypertrophy of ligamentum flavum in lumbar spine stenosis is associated with increased miR-155 level. Dis Markers. 2014;2014:786543.

6. Wang B, Gao C, Zhang P, et al. The increased motion of lumbar induces ligamentum flavum hypertrophy in a rat model. BMC Musculoskelet Disord. 2021;1(22):334

7. Zuo H, Cattani-Cavalieri I, Musheshe N, et al. Phosphodiesterases as therapeutic targets for respiratory diseases. Pharmacol Ther. 2019;197:225-42.

8. Ding $\mathrm{H}$, Bai $\mathrm{F}, \mathrm{CaO} \mathrm{H}$, et al. PDE/CAMP/Epac/C/EBP- $\beta$ signaling Cascade regulates mitochondria biogenesis of tubular epithelial cells in renal fibrosis. Antioxid Redox Signal. 2018;7(29):637-52.

9. Togo S, Liu X, Wang $X$, et al. PDE4 inhibitors roflumilast and rolipram augment PGE2 inhibition of TGF-\{beta\}1-stimulated fibroblasts. Am J Phys Lung Cell Mol Phys. 2009;6(296):L959-69.

10. Bender A, Beavo J. Cyclic nucleotide phosphodiesterases: molecular regulation to clinical use. Pharmacol Rev. 2006;3(58):488-520.

11. Essam R, Ahmed L, Abdelsalam R, et al. Phosphodiestrase-1 and 4 inhibitors ameliorate liver fibrosis in rats: modulation of CAMP/CREB/TLR4 inflammatory and fibrogenic pathways. Life Sci. 2019;222:245-54.

12. Takeda H, Nagai S, Ikeda D, et al. Collagen profiling of ligamentum flavum in patients with lumbar spinal canal stenosis. J Orthop Sci. 2020. https:// doi.org/10.1016/j.jos.2020.06.006.

13. Sun $\mathrm{C}$, Zhang $H$, Wang $X$, et al. Ligamentum flavum fibrosis and hypertrophy: molecular pathways, cellular mechanisms, and future directions FASEB J. 2020;8(34):9854-68.

14. Schiller M, Verrecchia F, Mauviel A. Cyclic adenosine 3',5'-monophosphate-elevating agents inhibit transforming growth factor-beta-induced
SMAD3/4-dependent transcription via a protein kinase A-dependent mechanism. Oncogene. 2003;55(22):8881-90.

15. Park J, Chang H, Lee J. Quantitative analysis of transforming growth factor-beta 1 in ligamentum flavum of lumbar spinal stenosis and disc herniation. Spine. 2001;21(26):E492-5.

16. Cutroneo K. TGF-beta-induced fibrosis and SMAD signaling: oligo decoys as natural therapeutics for inhibition of tissue fibrosis and scarring. Wound Repair Regen. 2007. https://doi.org/10.1111/j.1524-475X.2007. 00226.xS54-60.

17. Ye S, Kwon WK, Bae T, et al. CCN5 reduces Ligamentum Flavum hypertrophy by modulating the TGF-beta pathway. J Orthop Res. 2019;12(37):2634-44

18. Zheng $Z$, Ao X, Li P, et al. CRLF1 is a key regulator in the Ligamentum Flavum hypertrophy. Front Cell Dev Biol. 2020;8:858.

19. Nakamura T, Okada T, Endo M, et al. Angiopoietin-like protein 2 promotes inflammatory conditions in the ligamentum flavum in the pathogenesis of lumbar spinal canal stenosis by activating interleukin- 6 expression. Eur Spine J. 2015:9(24):2001-9.

20. Holm T, Habashi J, Doyle J, et al. Noncanonical TGF $\beta$ signaling contributes to aortic aneurysm progression in Marfan syndrome mice. Science. 2011;6027(332):358-61.

21. Griggs L, Hassan N, Malik R, et al. Fibronectin fibrils regulate TGF- $\beta 1$ induced epithelial-Mesenchymal transition. Matrix Biol. 2017. https://doi. org/10.1016/j.matbio.2017.01.001157-175.

22. Hur J, Bae T, Ye S, et al. Myofibroblast in the ligamentum flavum hypertrophic activity. Eur Spine J. 2017;8(26):2021-30.

23. Frangogiannis $N$. Transforming growth factor- $\beta$ in tissue fibrosis. J Exp Med. 2020;3(217):e20190103.

24. Kim S, Kim Y, Kim J, et al. Activation of ERK1/2-mTORC1-NOX4 mediates TGF- $\beta 1$-induced epithelial-mesenchymal transition and fibrosis in retinal pigment epithelial cells. Biochem Biophys Res Commun. 2020;3(529):747-52

\section{Publisher's Note}

Springer Nature remains neutral with regard to jurisdictional claims in published maps and institutional affiliations.
Ready to submit your research? Choose BMC and benefit from:

- fast, convenient online submission

- thorough peer review by experienced researchers in your field

- rapid publication on acceptance

- support for research data, including large and complex data types

- gold Open Access which fosters wider collaboration and increased citations

- maximum visibility for your research: over $100 \mathrm{M}$ website views per year

At $\mathrm{BMC}$, research is always in progress.

Learn more biomedcentral.com/submissions 